Rochester Institute of Technology

RIT Scholar Works

Presentations and other scholarship

Faculty \& Staff Scholarship

$12-1-2018$

\title{
Providing First Responders with Real-Time Status of Cellular Networks During a Disaster
}

\author{
Clark Hochgraf \\ Rochester Institute of Technology \\ Joseph Nygate \\ Rochester Institute of Technology \\ Miguel Bazdresch \\ Rochester Institute of Technology \\ Mark Indelicato \\ Rochester Institute of Technology \\ William P. Johnson \\ Rochester Institute of Technology
}

See next page for additional authors

Follow this and additional works at: https://scholarworks.rit.edu/other

\section{Recommended Citation}

C. Hochgraf, J. Nygate, M. Bazdresch, M. Indelicato, W. P. Johnson and R. E. Reyes, "Providing first responders with real-time status of cellular networks during a disaster," 2018 IEEE International Symposium on Technologies for Homeland Security (HST), Woburn, MA, 2018, pp. 1-4, doi: 10.1109/ THS.2018.8574145.

This Conference Paper is brought to you for free and open access by the Faculty \& Staff Scholarship at RIT Scholar Works. It has been accepted for inclusion in Presentations and other scholarship by an authorized administrator of RIT Scholar Works. For more information, please contact ritscholarworks@rit.edu. 


\section{Authors}

Clark Hochgraf, Joseph Nygate, Miguel Bazdresch, Mark Indelicato, William P. Johnson, and Romel Espinosa Reyes 


\section{Providing first responders with real-time status of cellular networks during a disaster}

\author{
Clark Hochgraf \\ College of Engineering \\ Technology \\ Rochester Institute of Technology \\ Rochester, NY, USA \\ cghiee@rit.edu
}

\author{
Joseph Nygate \\ College of Engineering \\ Technology \\ Rochester Institute of Technology \\ Rochester, NY, USA \\ janiee@rit.edu
}

William P. Johnson

College of Engineering

Technology

Rochester Institute of Technology

Rochester, NY, USA

wpjiee@rit.edu

\author{
Miguel Bazdresch \\ College of Engineering \\ Technology \\ Rochester Institute of Technology \\ Rochester, NY, USA \\ mxbiee@rit.edu
}

\author{
Romel Espinosa Reyes \\ Dept. of ICT Policies \\ Ministry of Telecommunications \\ and Information \\ Quito, Ecuador \\ romel.espinosa@mintel.gob.ec
}

\author{
Mark Indelicato \\ College of Engineering \\ Technology \\ Rochester Institute of Technology \\ Rochester, NY, USA \\ mjiiee@rit.edu
}

\begin{abstract}
Existing systems for reporting cellular outages do not provide adequate geographical granularity and do not provide a real-time view of the state of the communication network. This work presents a system for real-time measurement of both coverage and quality of service via crowdsourced measurements from consumer and first responder phones. Baseline coverage and quality of service data is collected prior to a major disaster. During a major disaster, real-time data is compared to baseline to identify areas of congestion or outages. Such information can be used by incident commanders to more effectively deploy resources during major disasters. Furthermore, such system state information can be used to support automatic deployment of temporary cellular network base stations, such as on drones.
\end{abstract}

Keywords-cellular networks, resilience, communications, disaster response

\section{INTRODUCTION}

Recent large-scale natural disasters, such as hurricanes Sandy, Harvey, and Maria, have caused significant damage to cellular communication networks, presenting a significant problem for first responders. Communications drive the response - they are the lifeblood. Nationally, it is estimated that $70 \%$ of 911 calls are made from cell phones [1]. Without cellular communications, affected individuals may not be able to report damage, fires, or request rescue or medical support.

More and more, first responder communications are occurring over the LTE cellular network. First responders are becoming reliant upon having access not only to voice communications, but SMS texts, video, and data connections to perform their job functions. Without data communications, situational awareness is decreased.

Knowing the state of the cellular communication system during a disaster and in the immediate aftermath would be of great value to first responders in deciding where to send search and rescue teams, evaluating where restoration services are needed, and knowing where communication between units is possible and not possible.

\section{Existing Methods For COMMUNiCATION NeTwORK ASSESSMENT}

There are several existing mechanisms for determining the state of cellular communication networks. These are examined and the limitations of these methods noted.

\section{A. NORS}

The network outage reporting system (NORS) requires cellular communication providers to notify the Federal Communications Commission (FCC) within 120 minutes of an outage and then to file an initial report within 72 hours of the initial event. The cell carriers report the number of user-minutes potentially affected by the outage, which has limited value as an indicator of the state of the communication network. NORS data doesn't provide timely information about what areas are affected and to what extent they are affected. The NORS system does not provide the on-going status of communication assets during a disaster and therefore isn't suitable for providing first responders the detailed, real-time coverage and quality of service (QoS) information they need. 


\section{B. Roll Call}

Roll Call is a system that can be deployed by FCC at the request of Federal Emergency Management Agency (FEMA) where FCC technicians are sent to the field to measure the radio spectrum before and after a disaster to determine if there are outages of wireless communication networks, broadcast radio, and TV. Data collection is done manually by a dedicated team using dedicated hardware and therefore cannot effectively provide coverage mapping over a large geographic region.

\section{DIRS}

Currently, when cellular networks are damaged in a major disaster, communication companies voluntarily provide mutual assistance under the Wireless Resiliency Cooperative Framework [2,3]. The Department of Homeland Security's National Communications System (NCS) developed the Disaster Information Reporting System (DIRS) to gather status information on communication assets from communication companies. DIRS covers wireless, wired, cable and broadcast services. The system was put into service in 2007 and is only activated during a disaster. Equipment status information is provided voluntarily by communication companies and is updated every 24 hours.

Due to confidentiality practices designed to protect commercially sensitive operational information from competitors, network asset status data is aggregated on county by county level basis and across all carriers. First responders are provided a count of how many cell towers there are in a county and how many are out of service in each county, but they are not provided with a map of where coverage is available within a county, nor are they able to find out what QoS is available at a given location (e.g. voice, text, video, data upload/download bandwidth). A sample DIRS coverage map from hurricane Harvey is shown in fig. 1.

\section{Percent Cell Sites Out-of-Service By County}

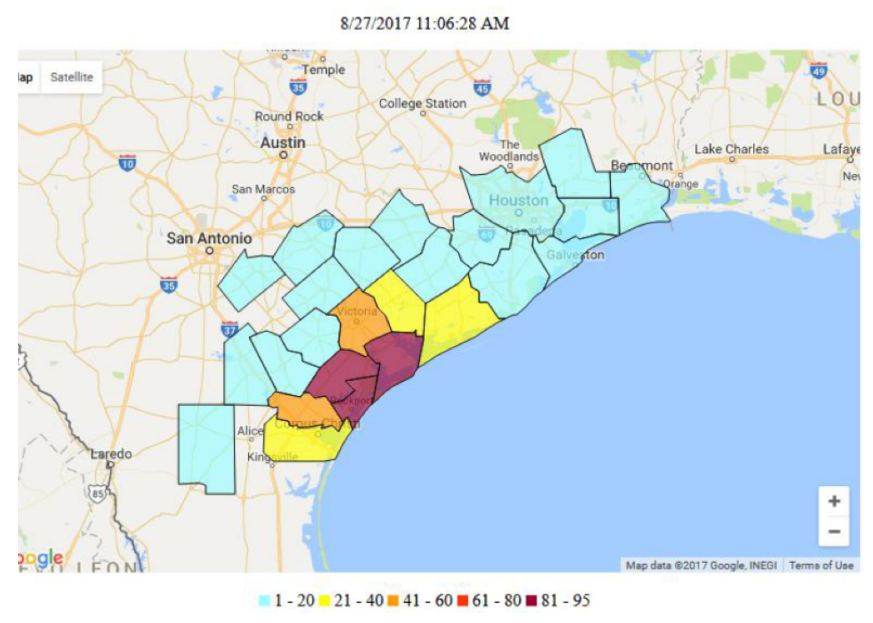

Fig. 1. Level of detail provided by DIRS system; colors indicate percent of cell sites out in a given county region, not actual coverage, coverage gaps or QoS. From [3]

This work was supported by the College of Engineering Technology, Rochester Institute of Technology.

\section{GAPS AND LIMITATIONS OF EXISTING APPROACHES}

The best currently available network information comes from DIRS, however there are very significant limitations on DIRS data. From a first responder perspective, DIRS data

1) is not sufficiently granular

2) doesn't provide coverage maps

3) doesn't provide QoS information

4 ) is updated too infrequently in dynamic situations (only once per 24 hours)

5 ) in raw form is too complex to be easily understood

DIRS tells which assets are operational but doesn't directly tell what areas are covered. This has to be inferred. More detailed DIRS data, such as a coverage heatmap, is available to FCC and NCS, but due to confidentiality restrictions this data isn't shared with first responders. As a result, the DIRS data is not sufficiently granular geographically nor temporally to produce actionable intelligence. For first responders, it doesn't communicate granular detail on where coverage exists or doesn't exist nor does it give information on the QoS available. Simply passing on raw DIRS reports to first responders is not a solution, as the data is so complex. A simpler digested version of data is needed.

\section{PROPOSED APPROACH}

An alternative to DIRS is direct measurement of the cellular network state using crowdsourced data from users via a smartphone app. A number of systems exist that provide alternative independent coverage maps for the various carriers [5-7]. These maps provide higher granularity of geographic coverage and recently have started collecting upload and download speed information. However, such apps are not necessarily directly suitable for measuring the network state during disasters.

\section{REQUIREMENTS FOR A FIRST RESPONDER CELL NETWORK STATUS MEASUREMENT SYSTEM}

Ideally, call takers at Public Safety Answering Points (PSAPs/911 centers) would have a real-time coverage map in front of them with clear indications of what QoS is available (e.g. voice, text, video, data upload/download bandwidth) at any location (street-level detail vs. county-level detail). That type of information should be available every day, not just on exercise days, so that operators are prepared to use that data effectively during disasters. First responders should also have the most recent real-time coverage maps available on their phones. The coverage information can change quickly as cell-site generators run out of fuel or tree clearing operations accidently cut fiber backhaul connections.

The challenges for crowdsourcing cell network coverage data using a smartphone app during a major disaster include:

1) obtaining coverage and QoS measurements with sufficient frequency to fill out a coverage map

2) minimizing the network bandwidth consumed by QoS measurements 
3) caching network coverage and QoS measurements on the phone until a backhaul path is available to share/update the coverage map and then merging data with the central coverage map/QoS database

4) minimizing battery consumption on the phone

5) validation of the data coming into the server to avoid the insertion of fake or misleading data

6) protection of individual identity and private information

7) cybersecurity

\section{SYSTEM ARCHITECTURE}

To address these needs, a custom system of software tools was architected and a prototype system built. The architecture allows ordinary smartphones to act as sensor nodes, geo-located measurements of LTE/3G/2G network parameters (e.g. signal strength, service type, etc.), as well as providing an economical direct measurement of upload and download speeds to compute QoS metrics.

The system architecture is shown in fig. 2. It utilizes a bandwidth test server to conduct upload/download tests to the smartphone. Test results are stored locally on the phone and uploaded to a central server where a composite coverage and QoS map is created. A secure web portal enables incident commanders to trigger an immediate bandwidth measurement

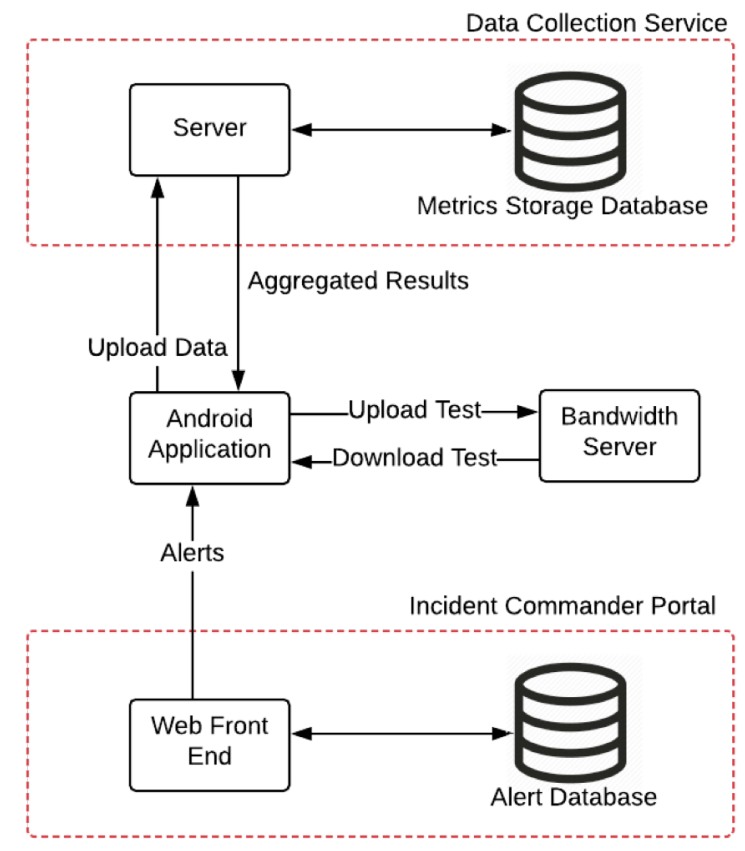

Fig. 2. System Architecture for crowdsourced measurement of cell network coverage and QoS.

test to get the current system connectivity and QoS status. This feature would only be used during a major event and is geographically limited to an area of interest defined by the incident commander. Otherwise, consumers might uninstall the app due to excessive battery consumption or data usage. In normal operation, the download and upload tests are triggered either by smartphone users or on a fixed time interval of 11 hours. The 11-hour interval causes measurements to be distributed uniformly through out the day, assuming the phone start-up time is randomly distributed through the hour across many users.

\section{SYSTEM PROTOTYPE RESULTS}

A sample QoS map based on measured download speeds is shown in fig. 3. Square regions in red have lower relative download speed compared to square regions in green. Regions that are not shaded either color have no data. The map data can be filtered by carrier and time interval. For example, the time interval can be selected as the last 15 minutes, last hour, last 4 hours, last day, week, month, or for all time measured.
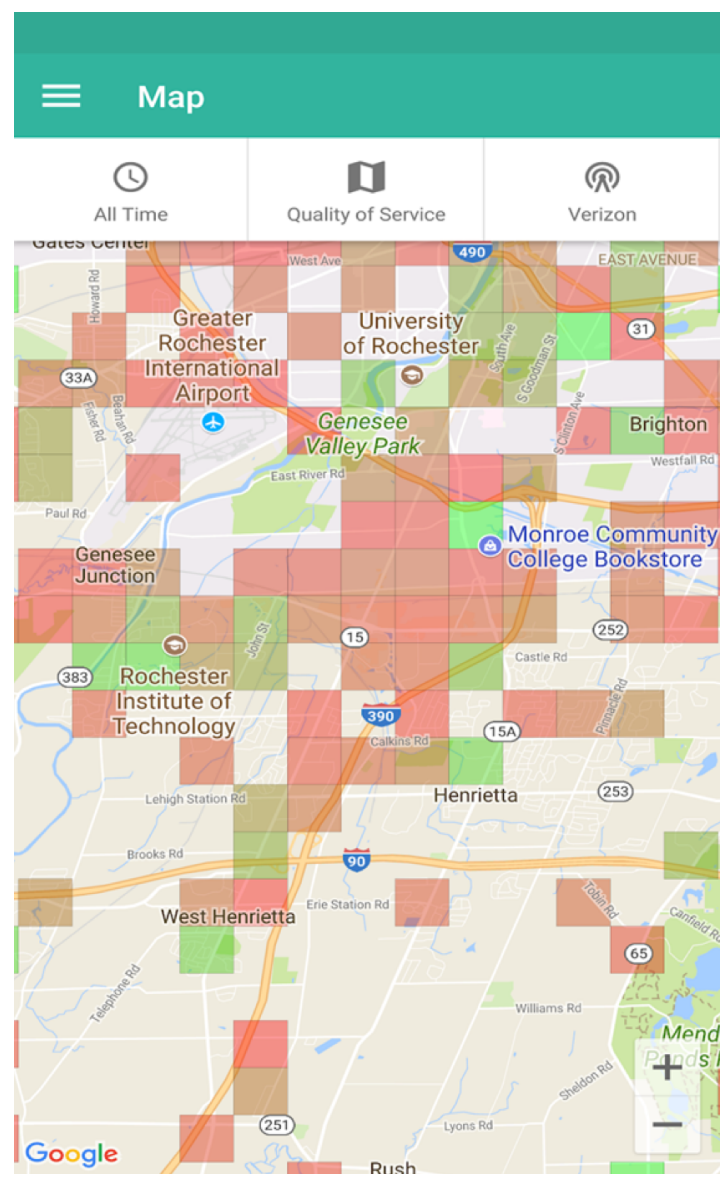

Fig. 3. Quality of service measurement map derived from crowdsourced data. Green indicates higher average download speeds. Note some areas have no data points (squares that are neither green nor red). Quality of service maps can be generated for the last hour, day or week.

\section{APPLICATION TO DEPLOYABLE SYSTEMS}

Comparing realtime measurements of cell coverage and QoS with baseline data allows the location of outages or communication bottlenecks to be identified. Incident commanders can combine this information with other situational information to prioritize where temporary cell tower assets e.g. Cell tower On Wheels systems (COWs) should be deployed. 
Ideally, the cell tower deployment location decision can be automated, reducing workload on the incident commander. For example, in the future, a network of drones carrying LTE base stations could automatically re-position themselves to balance load across multiple drones and close coverage gaps as identified by the QoS measurement system.

Employing the QoS measurement system on drones or COWs involves careful consideration of a number of factors [9], including the energy demands and bandwidth usage required to conduct tests as well as how to merge coverage databases between drones, user equipment and the central coverage map.

Bandwidth and energy usage can be minimized by careful choice of the measurement update frequency and size of test download package. Exploratory work is underway to infer the current download and upload speed from existing data traffic as well as how to combine the passive and active bandwidth measurement methods.

Sharing and merging coverage/QoS data between nodes is also a challenge. In a distributed network of drone LTE stations, communication between individual drone units, between drones and user equipment, and between the central QoS database (on the backhaul network) will likely be intermittent. Each node in the network (e.g. drone, user equipment, fixed network) has partial information about the communication network's system state. Furthermore, the system state is continuously changing so information shared between any of the nodes will quickly become outdated.

Each node may be generating information on the system state, receiving state information from other nodes and passing along state information from other nodes. How long to keep such information before it is deemed stale and what information to pass along are key design decisions. Further work includes examining these issues in the context of self-organizing and self- deploying networks of mobile LTE base stations, whether deployed on drones or transportable systems.

\section{CONCLUSION}

This paper presents a method for independently measuring the state of the cellular communication network in real time using crowdsourced measurements from users' smartphones. The method does not rely on information from carriers. Both cellular coverage and QoS can be determined. The system is applicable to disaster communications as it provides high spatial resolution and faster temporal updates than exising reporting systems including NORS, DIRS, and Roll Call. Future work includes development of a scaled version suitable for deployment in a highly mobile network of deployable LTE base stations e.g. LTE base stations flying on drones.

\section{REFERENCES}

[1] https://www.fcc.gov/consumers/guides/911-wireless-services, accessed May $1,2018$.

[2] Ex Parte Presentation to FCC Secretary Marlene H. Dortch, Improving Resiliency, Reliability and Continuity of Mobile Wireless Communications Networks, PS Docket Nos. 13-239 and 11-60, April 27, 2016.

[3] https://www.fcc.gov/wireless-resiliency-cooperative-framework, accessed May 1, 2018

[4] FCC Communications Status Report for Areas Impacted by Tropical Storm Harvey, August 27, 2017.

[5] G. Bartoli, R. Fantacci, F. Gei, D. Marabissi, and L. Micciullo, "A novel emergency management platform for smart public safety," Int. J. Commun. Syst., vol. 28, no. 5, pp. 928-943, March 2015.

[6] http://webcoveragemap.rootmetrics.com/us, accessed May 1, 2018.

[7] https://opensignal.com/networks, accessed May 1, 2018.

[8] https://www.sensorly.com/, accessed May 1, 2018.

[9] H. Wang, G. Ding, F. Gao, J. Chen, J. Wang and L. Wang, "Power Control in UAV-Supported Ultra Dense Networks: Communications, Caching, and Energy Transfer," in IEEE Communications Magazine, vol. 56, no. 6, pp. 28-34, June 2018. doi: 10.1109/MCOM.2018.1700431 